\title{
Profiling the mouse brain endothelial transcriptome in health and disease models reveals a core blood-brain barrier dysfunction module
}

\author{
Roeben Nocon Munji ${ }^{1,{ }^{*}}$, Allison Luen Soung ${ }^{1,{ }^{*}}$, Geoffrey Aaron Weiner ${ }^{1}$, Fabien Sohet ${ }^{1}$, \\ Bridgette Deanne Semple ${ }^{2}$, Alpa Trivedi ${ }^{2}$, Kayleen Gimlin², Masakazu Kotoda ${ }^{3}$, Masaaki \\ Korai $^{3}$, Sidar Aydin ${ }^{1}$, Austin Batugal ${ }^{1}$, Anne Christelle Cabangcala ${ }^{1}$, Patrick Georg \\ Schupp ${ }^{2}$, Michael Clark Oldham ${ }^{2}$, Tomoki Hashimoto ${ }^{3}$, Linda J. Noble-Haeusslein ${ }^{2}$, Richard $^{2}$ \\ Daneman ${ }^{1,+}$ \\ ${ }^{1}$ Departments of Pharmacology and Neurosciences, University of California, San Diego. San \\ Diego, CA, 92093 USA \\ ${ }^{2}$ Department of Neurological Surgery, University of California, San Francisco San Francisco, CA, \\ 94143 USA \\ ${ }^{3}$ Department of Neurosurgery and Neurobiology, Barrow Aneurysm and AVM Research Center, \\ Barrow Neurological Institute, Phoenix, AZ 85013 USA
}

\begin{abstract}
Blood vessels in the central nervous system (CNS) form a specialized and critical structure, the blood-brain barrier (BBB). We present a resource to understand the molecular mechanisms that regulate BBB function in health and dysfunction during disease. Using endothelial cell enrichment and RNA sequencing, we analyzed the gene expression of endothelial cells in mice, comparing brain endothelial cells to peripheral endothelial cells. We also assessed the regulation of CNS
\end{abstract}

\footnotetext{
Users may view, print, copy, and download text and data-mine the content in such documents, for the purposes of academic research, subject always to the full Conditions of use:http://www.nature.com/authors/editorial_policies/license.html\#terms

${ }^{+}$Correspondence to: Richard Daneman, UCSD, Departments of Pharmacology and Neuroscience, 9500 Gilman Dr, BSB3092, La Jolla, CA, 92093-0636, Rdaneman@ucsd.edu.

These authors contributed equally

Author Contributions

R.D. designed and participated in the analysis of all experiments. R.N.M. designed the seizure and Wnt signaling studies, performed cell purification of health and Wnt signaling models, participated in the analysis of health, disease and Wnt signaling studies, and writing of the manuscript. A.L.S. performed the endothelial cell purification and analysis of health and disease models. G.A.W. performed the alignment and normalization of RNA sequencing data. P.G.S. and M.C.O. designed and performed the gene coexpression analysis. F.S. participated in the design and analysis of the EAE model. L.J.N.-H., B.D.S., A.T. and K.G. participated in the design and analysis of all TBI experiments. T.H., M.Korai and M.Kotoda participated in the design and analysis of all MCAO experiments. S.A. participated in the design and analysis of the Wnt signaling study. A.B. and A.C.C. participated in the design and analysis of disease models.

Accession codes

GEO: The cerebrovascular response to injury and disease-GSE95401, Gene expression profiles of liver and lung endothelial cells during normal and upregulation of Wnt/beta-catenin signaling-GSE95201.

Competing Interests

The authors declare no competing interests.

Data Availability

The data that support the findings of this study is available from the corresponding author upon request. RNA sequencing files were deposited in the Gene Expression Omnibus repository and are available to use.

Specific experimental details are also organized in the accompanying Nature Research Life Science Reporting Summary.
} 
endothelial gene expression in models of stroke, multiple sclerosis, traumatic brain injury and seizures, each having profound BBB disruption. We found that although each is caused by a distinct trigger, they exhibit strikingly similar endothelial gene expression changes during BBB disruption, comprising a core BBB-dysfunction module that shifts the CNS endothelial cells into a peripheral endothelial cell-like state. The identification of a common pathway for BBB dysfunction suggests that targeting therapeutic agents to limit it may be effective across multiple neurological disorders.

The blood vessels in the central nervous system (CNS) possess a series of unique properties, together termed the blood-brain barrier (BBB), that tightly regulate the movement of ions, molecules and cells between the blood and the neural tissue1,2. Many of these BBB properties are mediated by the endothelial cells that line the blood vessels. In contrast to those in non-neural tissues, CNS endothelial cells have specialized tight junction structures that maintain a high electrical resistance paracellular barrier, low rates of transcytosis and lack of fenestra creating a transcellular barrier, distinct transport properties that efflux potential toxins and deliver specific nutrients, and low levels of leukocyte adhesion molecules that limit CNS immune surveillance1-3. These properties are regulated by interactions between the endothelial cells with the CNS microenvironment4,5, including neural progenitors, pericytes and astrocytes $4,6-9$. The ability of the BBB to tightly regulate the microenvironment of the CNS is critical for the proper neuronal function and to protect neural tissue from toxins, pathogens and other potentially harmful agents.

BBB disruption has been observed in human patients and mouse models of many different neurological diseases including stroke, multiple sclerosis (MS), traumatic brain injury (TBI), epilepsy, cancer, infection and neurodegenerative diseases1,2. The disruption of the BBB can include a loss of tight junction integrity, increase in transcytosis, alterations in transport properties and increases in the expression of leukocyte adhesion molecules. These changes in the BBB result in CNS ion dysregulation, edema and immune infiltration, which can lead to neuronal dysfunction, damage and degeneration. Despite its importance in disease, many questions still remain. What are the molecular mechanisms that lead to BBB dysfunction in each disease? Is disruption of the BBB mediated by the same or different mechanisms in different neurological diseases? How is the BBB repaired? Is BBB dysfunction helpful in wound healing or harmful, initiating neuronal damage?

Here we have used endothelial cell enrichment followed by RNA sequencing to generate a resource to understand BBB gene expression in health and disease in mice. In health we enriched for endothelial cells from different organs including the brain, heart, kidney, lung, and liver, and sequenced the RNA to generate a BBB-specific gene expression profile. We further used four different disease models including a middle cerebral artery occlusion (MCAO) model of stroke, an experimental autoimmune encephalomyelitis (EAE) model of MS, a cortical impact model of pediatric TBI, and a kainic acid model of seizure, each with distinct temporal and spatial patterns of BBB dysfunction and neuroinflammation. For each disease model, we enriched for the endothelial cells and performed RNA sequencing from three timepoints to identify the endothelial gene expression changes following each of the different triggers. This RNA sequencing database provides a resource for understanding the 
transcriptional profiles of CNS endothelial cells during health and disease. We found that, although each of the disease models has a unique trigger, they each lead to remarkably similar transcriptional changes to the BBB, suggesting a common mechanism for BBB dysfunction throughout different neurological disorders.

\section{RESULTS}

\section{The blood-brain barrier in health}

Transcriptional profiling of different vascular beds-Rosa-tdTomato; VE-Cadherin$\mathrm{Cre}_{\text {ERT2 }}$ mice were generated to enable tamoxifen-inducible expression of tdTomato in endothelial cells. One week following tamoxifen injections in adults, tdTomato fluorescence could be visualized in blood vessels in brain, spinal cord, heart, kidney, lung and liver (Fig. 1). The tdTomato co-localized with $\mathrm{CD} 31_{+}$endothelial cells in each of the tissues, and did not co-localize with immune cells, pericytes, neuronal or glial cell markers (Fig. 1, data not shown). We enriched tdTomato t $_{+}$endothelial cells from brain, heart, kidney, lung and liver using FACS, isolated the RNA and performed RNA sequencing, as well as analyzing whole brain homogenates. Because brain mural cells adhere tightly to the endothelial cells, we added a second set of brain samples with an extra collagenase/dispase digestion step. We termed the first set Brain Vascular, as it contains endothelial cells with some adherent mural cells, and the second set Brain Endothelial, as the mural cells are further depleted. Reads were mapped onto the ensembl genome. The brain vascular and brain endothelial cell samples showed high levels of RNA from endothelial cell genes with minimal levels of RNA from neuronal and glial genes. In the Brain Vascular sample there was a small but present level of mural cell genes estimated to be $<2.0 \%$ of the RNA whereas the Brain Endothelial sample contained $<0.05 \%$ mural cell RNA (Supplementary Fig. 1). The complete data set can be found in Supplementary File 1. Brain mural cell genes could thus be identified as genes enriched in the Brain Vascular compared to the Brain Endothelial sample (Supplementary File 2).

BBB-enriched transcriptome-In Supplementary File 3, we list all the BBB-enriched genes ( $>5$ counts per million $[\mathrm{cpm}]$ in brain endothelial cells, and at least two-fold $(\log 2>1.000)$ and P-value $<0.05$ enriched in brain endothelial cells compared to endothelial cells of each peripheral organ), and in Supplementary Table 1, we list the top 50 BBBenriched genes (most enriched in the brain endothelial cells compared to the heart, kidney, lung and liver endothelial cell samples and whole brain samples). We utilized the DAVID Bioinformatics functional annotation tool to identify signaling pathways, metabolic pathways and protein interactions enriched at the BBB. This identified Wnt/beta-catenin related pathways, different transport mechanisms, and amino acid metabolism as key BBBenriched pathways. Wnt/beta-catenin signaling has been identified as a key regulator of CNS-specific angiogenesis, BBB induction and maintenance10-16, and this data-set identified BBB-enriched Wnt mediators including Lef1, Fzd3, Notum, Apcdd1, Axin2, dixdc1 and Tnfrsf19. This dataset identified BBB-enriched components of tight junctions (TJ) (Supplementary File 4, Supplementary Table 2), transporters (Supplementary Table 3), and additional BBB-enriched functions including extracellular matrix, metabolic programs and transcription factors (see Supplementary Results and Discussion). 
Peripheral endothelial-enriched transcriptome-This resource also identified genes enriched in the peripheral endothelial cells compared to brain endothelial cells, as well as genes enriched in each specific vascular bed. In Supplementary File 5 we list all the peripheral endothelial-enriched genes ( $\mathrm{cpm}>5$ in all of the peripheral endothelial samples, with a $\log 2$ ratio $>1.00$ and $\mathrm{P}$-value $<0.05$ for at least three of the peripheral endothelial samples compared to the brain endothelial samples). In Supplementary Table 1, we list the 50 most peripheral enriched genes. Pathways mediating the immune response including leukocyte migration, toll-like receptor signaling, chemokine signaling and antigen presentation are enriched in peripheral endothelial cells compared to brain endothelial cells. Several of these genes are known to mediate the function of peripheral endothelial cells, including Plvap, which regulates transcytosis17-19, and Sele, Selp, Vcam and Icam1, which mediate leukocyte adhesion20-30. Several Hox genes, including Hoxa5 and Hoxb4, are greatly enriched in the peripheral endothelial cells compared to the brain endothelial cells that may indicate a rostral-caudal axis identity for vasculature. In addition there are many instances of a peripheral-enriched gene with a corresponding BBB-enriched family member. This includes aquaporins, annexins, and semaphorins.

\section{Cerebrovascular transcriptional response to disease}

BBB dysfunction in disease models-Next we examined the molecular changes to CNS endothelial cells following four different mouse models of neurological diseases and injuries: 1) Kainic acid (KA) model of seizure, 2) experimental autoimmune encephalomyelitis (EAE) model of MS, 3) middle cerebral artery occlusion (MCAO) model of stroke, and 4) a focal cortical impact model of pediatric TBI. Each model is elicited by a different trigger (neuronal overactivity/seizure; auto-inflammation/EAE; ischemia/stroke and mechanical injury/TBI), but all lead to BBB dysfunction and neuroinflammation.

For each model we examined BBB dysfunction, using a transcardiac perfusion of a molecular tracer (sulfo-NHS-biotin, 500Da) at three timepoints: acute, subacute and chronic. These timepoints differed based on the course of the disease (see methods), but in general the acute timepoint was within a day of the onset, the subacute time point was two days following the acute, and the chronic was 1-month post onset.

For the KA model of seizure, there was negligible vascular leakage at the acute timepoint during the active seizures, whereas at the subacute timepoint vascular leakage could be visualized throughout the temporal lobe, and to a lesser extent the hippocampus. At the chronic timepoint there was minimal vascular leakage (Fig. 2A,E; Supplementary Fig. 2). For the EAE model, there was localized BBB leakage at sites of active lesions throughout the spinal cord at the acute and even greater leakage at the subacute timepoint. At the chronic timepoint there were sporadic points of leakage that localized to a subset of the lesions (Fig. 2B,F; Supplementary Fig. 2). For the MCAO model of stroke, there were rare small focal points of vascular leakage on the ipsilateral hemisphere at the acute timepoint, which appeared to result from isolated vessels with a leaky BBB. the subacute timepoint there was a large discrete region with clear boundaries within the ipsilateral hemisphere that showed vascular leakage. At the chronic timepoint, there was a much smaller region of BBB leakage in a discrete scar (Figure 2C,G; Supplementary Fig. 2). For TBI, leakage occurred 
preferentially in the cortex adjacent to the site of impact at the acute timepoint. At the subacute time point, the pattern of leakage was more robust, extending into the neighboring cortex. The chronic timepoint was characterized by prominent cavitation with minimal and sporadic BBB leakage. (Figure 2D,H; Supplementary Fig. 2). We found that the BBB in all four of the disease models exhibited increased permeability to large endogenous molecules, including fibrinogen and $\mathrm{IgG}$, at the subacute timepoint compared to their corresponding controls (Supplementary Fig. 3-4). Although we observed only a few hypertrophic vessels with disorganized Claudin 5 in the seizure model, there were numerous hypertrophic vessels with disorganized Claudin 5 in EAE, stroke and TBI (Supplementary Fig. 5). For each disease we also examined the expression of the inflammatory marker CD45 in the CNS, and found robust inflammation, peaking at the subacute timepoint (Fig. 2, see Supplementary Results and Discussion).

We then used endothelial cell enrichment and RNA sequencing to analyze the gene expression for each disease at the three timepoints. In each case the area of CNS corresponding to the inflamed region was dissected for endothelial cell purification. In addition, we used immune-lineage negative selection to limit immune cell contamination. The complete data set of gene expression is presented in Supplementary File 1. In addition, we performed co-expression analysis to identify modules of genes, named by different colors, that followed statistically similar patterns of expression over all the health and disease samples. In Supplementary File 1, Tab 4 we present the association of each gene with each module and report the strongest associations after correcting for multiple comparisons (Bonferroni correction [column F] and False Discovery Rate [column G]). This analysis revealed that many of the BBB-enriched genes affiliate with the red or dark grey modules, while many peripheral-enriched genes affiliate with the black, blue, salmon, pink or tan modules.

Comparisons of gene changes during each disease-To compare the changes in gene expression between each disease we defined the number of up-regulated genes ( $\log 2$ ratio>1.000; $\mathrm{P}$-Value $<0.05$, minimum mean value of 5 counts per million [cpm] in the disease samples) and down-regulated genes ( $\log 2$ ratio $<-0.800$; P-Value $<0.05$, minimum mean value of $5 \mathrm{cpm}$ in the healthy samples) at each timepoint for each disease and determined the overlap of gene changes between timepoints for each disease and between different diseases within a timepoint.

In Figure 3A for each disease the overlap of gene changes between timepoints are shown with Venn diagrams. In seizure and TBI models, the most unique changes are observed at the acute timepoint, whereas for EAE and MCAO the most unique changes are observed at the subacute timepoint. For seizure, stroke and TBI models, the most overlap of the changes occurred between the acute and subacute timepoints. For EAE the most overlap occurred between the subacute and chronic timepoints. These differences likely indicate the distinctions between the severity of the initial insult in the seizure, stroke, and TBI models compared to the buildup of inflammation in the EAE model of MS.

In Figure 3B and 3C the overlap of gene changes between diseases is highlighted. The largest number of overlapping gene changes are observed at the subacute timepoints with the 
majority of genes altered, especially up-regulated genes, in seizure, stroke and TBI models are also changed in the other disease models. The acute timepoint, on the other hand, showed many disease-specific changes as there were largely non-overlapping data-sets between diseases. Therefore, the triggers for each disease initially elicit different transcriptional changes at the $\mathrm{BBB}$, but then converge on similar changes at the subacute timepoint when the BBB is most leaky. At the chronic timepoint the least number of changes were observed for seizure, stroke and TBI models.

We determined how the BBB-enriched and peripheral endothelial-enriched data sets responded to each of the diseases (Figure 3D,E). The BBB-enriched transcriptome was down-regulated during each of the diseases, reaching the lowest value at the acute TBI, and subacute seizure, EAE and stroke timepoints. The peripheral endothelial-enriched genes were up-regulated, peaking at acute TBI, and subacute seizure, EAE and stroke timepoints. Therefore CNS endothelial cells take on a peripheral endothelial cell gene expression profile during BBB dysfunction. Using DAVID Bioinformatics we identified the signaling and biological pathways altered in the CNS endothelial cells at each timepoint in each disease (Fig. 4, see Supplementary Results and Discussion).

Common changes among diseases-The subacute timepoint, when the most severe BBB dysfunction was observed, had the most common gene changes between each disease with 54 genes up-regulated in all four diseases and 136 genes up-regulated in at least three of the four diseases (Fig. 5A, Supplementary File 6, Supplementary Table 4). This is most striking for the TBI and stroke models. For TBI, $61.2 \%$ of the upregulated genes at the subacute timepoint are upregulated in at least three of the four diseases, and $85.2 \%$ of the genes are upregulated in at least one other disease. For the stroke model, $52.1 \%$ of the upregulated genes at the subacute timepoint are upregulated in at least three of the four diseases, and $82.2 \%$ of the genes are upregulated in at least one other disease. For the seizure model, $22.9 \%$ of the upregulated genes at the subacute timepoint are upregulated in at least three of the four diseases, and 54.4\% of the genes are upregulated in at least one other disease. The EAE model showed the most changes, and also the most unique changes. In this model, $16.4 \%$ of the upregulated genes at the subacute timepoint are upregulated in at least three of the four diseases, and $42.1 \%$ of the genes are upregulated in at least one other disease. All four disease models led to remarkably similar gene expression changes at the $\mathrm{BBB}$, and this resource has identified a common BBB dysfunction module that appears to be up-regulated in CNS endothelial cells regardless of the trigger. On average the BBB dysfunction module peaked at the acute timepoint in the TBI model and the subacute timepoint in seizure, EAE, and stroke models (Fig. 5B). The genes then returned to baseline expression at the chronic timepoint in the seizure, stroke and TBI models, but remained elevated in the EAE model (Fig. 5B).

We determined the expression of these BBB dysfunction genes during health by determining the expression of each gene in the brain and each peripheral endothelial cell sample. On average, the BBB-dysfunction module genes were enriched in each of the peripheral vascular beds when compared to the brain (Fig. 5C), and 68 of the 138 genes were identified as peripheral-enriched (see Supplementary File 6, column FQ). Therefore in each disease model, CNS endothelial cells take on a 'peripheral' endothelial gene expression pattern. 
KEGG and GO term pathways in this BBB dysfunction module include those involved in cell division, blood vessel development, inflammatory response, wound healing, leukocyte migration, and focal adhesion, highlighting a role for angiogenesis and inflammation in BBB dysfunction (Fig. 5D). This core BBB dysfunction module of 136 genes includes several genes that have been identified to play a role in BBB dysfunction in different diseases. These include genes that regulate leukocyte trafficking (Sele, Selp), and proteolytic cleavage of ECM (Mmp14). In addition, multiple members of several gene families were upregulated: extracellular proteases of the Serpin family (Serpine1, Serping1), Adams and Adamts families (Adam12, Adam19, Adamts4, Adamts8), collagens (Colla1, Colla2, Col3a1, Col5a1, Col5a2, Col12a1), centromere proteins (Cenpe, Cenpf), Igf binding proteins (Igfbp4, Igfbp5), kinesins (Kif11, Kif15, Kif20b), lysyl oxidases (lox, Lox12, Lox3), sulfatases (Sulf1, Sulf2), thrombospondins (Thbs1, Thbs2) and pleckstrin domain containing genes (Plekho1, Plekho2). One of the main categories of genes in the BBB dysfunction module were extracellular matrix proteins (Col1a1, Col1a2, Col3a1, Lamb1) and modulators of the extracellular matrix including extracellular proteases (Adam12, Adam19, Adamts4, Adamts9, Mmp14), extracellular protease inhibitors (Serpine1, Serping1), and matrix crosslinkers (Lox, Lox12, Lox3). Using immunohistochemistry we validated that indeed many of these matrix proteins are increased along vessels in each disease (Supplementary Fig. 6-7).

The BBB dysfunction module genes could be segregated into three patterns based on the temporal regulation in the diseases (Fig. 5E). Group 1 reached peak up-regulation in the acute phase of many of the diseases and consisted of genes that included molecules involved in inflammation (Sele, Timp) and extracellular proteases (Adamts4, Adamts8). Group 2 peaked at the subacute phase in most of the disease models and included inflammation (Selp, Darc) and cell cycle (Ccna2, Cenpe, Mki67) genes. Group 3 peaked at early timepoints in seizure, stroke and TBI models, but continued to increase in EAE, and included ECM genes (Col3al) and inhibitors of angiogenesis (Thsb1, Thsb2).

In the co-expression analysis the BBB-dysfunction module genes were found in different modules including the black, pink, salmon and steelblue modules (Supplementary File 1, tab 4). Interestingly, while the black, pink and salmon modules were consistent with peripheralendothelial cell genes, the steelblue module consisted of many genes expressed by fibroblasts. This could suggest that there is an endothelial-mesenchymal transition occurring during disease as has been hypothesized during BBB dysfunction31,32, or could occur if endothelial cells engulf fibroblast RNA particles or by fibroblast contamination in the endothelial samples. In addition to the common changes found at the subacute timepoint, there were common changes found at the acute and chronic timepoints (see Supplementary Results and Discussion).

Changes unique to each disease-The most robust disease-unique changes occurred in the seizure model at the acute timepoint, during seizures and prior to BBB dysfunction and inflammation. Interestingly many solute carrier transporters (65 genes) and ABCtransporters (11 genes) were down-regulated during the seizures corresponding with an upregulation of the glucose transporter Glut1 (Slc2a1), suggesting that the BBB modulates its 
gene expression in response to neuronal activity, perhaps to focus on glucose transport to meet the heavy energetic demands of the seizures.

The disease with the most unique changes at the subacute timepoint was EAE. Interestingly, many of these unique changes in EAE were family members of genes within the core-BBB dysfunction module, including additional members of the following families: Serpin (Serpine1 and Serping1 are up-regulated in at least 3 diseases while Serpina3f, Serpina3g, Serpina3i, serpina3n, serpinb1a, serpinb9, serpinb9b are up-regulated just in EAE), MMP (Mmp14 and Mmp23), Adam (Adam12/Adam19 and Adam9), Adamts (Adamts4/Adamts8 and Adamts2/Adamts5), centromere proteins (Cenpe/Cenpf, and Cenpa/Cenpi), and kinesins (Kif11/Kif15/Kif20b and Kif18b). This suggests that there is a core BBB dysfunction pathway, and that additional family members are recruited the more severe the dysfunction. There were many additional unique changes in each disease (see Supplementary Results and Discussion).

\section{Regulation of endothelial gene expression by Wnt/beta-catenin signaling}

Wnt/beta-catenin signaling has been identified as a key regulator of CNS angiogenesis, BBB formation and BBB maintenance inducing tight junction, solute transporter and efflux transporter expression, and repressing Plvap expression10-16. We utilized a mouse model (Rosa-Bcat-GOF; VE-Cadherin-Cre ERT2 $_{2}$ mice) to express constitutively active beta-catenin in endothelial cells, and then used endothelial enrichment and RNA sequencing to determine which genes activated beta-catenin is sufficient to regulate in peripheral endothelial cells.

In Supplementary File 7 we present the RNA sequencing data comparing expression in liver and lung endothelial cells from activated beta-catenin (Rosa-Bcat-GOF; VE-Cadherin$\mathrm{Cre}_{\text {ERT2}}$ ) and control mice (Rosa-Bcat-GOF). In Supplementary Table 5 we present the top 20 genes that were up- or down-regulated in liver and lung endothelial cells by activated beta-catenin. Activated beta-catenin had a greater effect on liver endothelial cells (882 genes altered $\mathrm{P}<0.05, \mathrm{cpm}>10$ in control or Bcat-GOF) than lung endothelial cells ( 257 genes altered $\mathrm{P}<0.05, \mathrm{cpm}>10$ in control or Bcat-GOF)(Fig. 6A). These findings suggest that the highly permeable liver endothelial cells are more responsive to $\mathrm{Wnt} /$ beta-catenin activation than the moderately permeable lung endothelial cells. More BBB-enriched genes were upregulated (34-liver, 12-lung) than down-regulated (5-liver, 5-lung) in the peripheral endothelial cells due to activation of beta-catenin, whereas more peripheral-enriched endothelial genes were down-regulated (92-liver, 28-lung) than up-regulated (50-liver, 23lung) (Fig. 6A,B). The up-regulated genes are expressed higher in healthy brain endothelial cells than peripheral endothelial cells (Fig. 6C). Therefore, activated beta-catenin caused peripheral endothelial cells to take on more of a brain endothelial gene expression profile.

Lastly, we examined how genes regulated by activated beta-catenin were changed in CNS endothelial cells in different disease models (Fig. 6D). We did not find any correlation between the beta-catenin activated gene changes and the changes observed in disease. Although the Wnt/beta-catenin pathway has been implicated in BBB formation and maintenance, our data suggest that loss of Wnt/beta-catenin signaling may not be the driving force behind the transcriptional changes that we observed in the different disease models. 


\section{Discussion}

We have utilized endothelial cell enrichment and RNA sequencing to understand the gene expression changes to the BBB in different neurological disease models that display BBB dysfunction. Although each of the mouse models has different triggers, we found similar gene expression changes to the BBB in each of the diseases at the subacute timepoint when the BBB was most dysfunctional. These data suggest that although the disparate triggers may initially have different effects on the vasculature, they converge on similar responses in the endothelial cells. This has important implications for the treatment of neurological diseases in which BBB dysfunction is a contributing factor, including epilepsy, MS, stroke and TBI, as it suggests that identifying therapeutics that limit BBB dysfunction in one of the diseases may lead to treatments for others. Increased BBB permeability can be both helpful, in allowing the entry of peripheral immune cells to aid in the clearance of debris and wound healing, but also detrimental, as it can lead to neuronal dysfunction, intracranial pressure increases and immune-mediated neuronal damage and degeneration. As with all inflammatory processes it is often the scale of the response that is critical, with small amounts being advantageous and large amounts detrimental. Therefore developing methods to modulate these pathways to control the timing, spatial distribution and amount of BBB dysfunction will be critical.

It is of particular importance to understand the role of each gene of the BBB-dysfunction module in regulating alterations in the properties of the BBB. These genes may have negative pathological consequences including leakage of the BBB, driving neuroinflammation and generating a fibrotic scar, or positive consequences including feedback mechanisms that are neuro-protective or for BBB repair. While many of the genes in this BBB-dysfunction module are novel, several have been identified to have key roles in the disease process. For instance, leukocyte adhesion molecules (Sele, Selp), chemokines (Ccl2), and TNF family members ( Tnfsf8) have been shown to drive inflammation and damage24,33-35. On the other hand, up-regulation of apelin ( $A p l n)$ has been found to be neuro-protective36-38.

It is also striking that a subset of this BBB-dysfunction module was still elevated at the chronic timepoint after BBB permeability had restored to baseline in many of the diseases. This suggests that a single trigger, such as a three hour KA induced seizure, can lead to long term changes to the vasculature that could potentially have consequences on the function of the brain. For instance, changes in transporters, signaling, or the extracellular matrix could alter the CNS microenvironment and have implications for neural circuit function. Persistent changes, such as tmem176a, slc1a3, slc6a13, slc7a11 or Tlr2, could potentially be used to identify and target therapeutics to a site of previous pathology.

Each disease also displayed unique brain endothelial transcriptional changes at each time point, likely reflecting the severity of the vascular insult, different inflammatory changes, amount of neuronal and glial cell death and activation, and differing repair processes in each disease. At the subacute and chronic timepoints, the largest numbers of changes were observed in the EAE model of MS. This is most likely due to the diversity, severity and length of inflammation in this model which engages both the innate and the adaptive 
immune systems as the neural tissue is invaded with CD4+ T-Cells, CD8+ T-Cells and BCells in addition to innate immune cells. This is reflected in the enhanced immune interacting gene changes in EAE including leukocyte adhesion molecules (Vcam1), histocompatibility loci, interferon induced genes, interleukin pathway genes, and complement pathway genes.

The most unique changes at the acute timepoint were observed in the KA induced seizure model, when the seizures were still pervasive but no inflammation or BBB leakage was observed. These unique changes are likely due to the increased metabolic demand in response to high levels of neuronal activity, as it appears the endothelial cells alter their transport properties to concentrate on delivery of glucose to the neural tissue. This suggests that the BBB is capable of dynamically altering its properties in response to neural activity.

Wnt/beta-catenin signaling is a key regulator CNS angiogenesis, BBB formation and maintenance, and has been specifically linked to the induction of solute transporters, tight junction proteins, efflux transporters and inhibition of transcytosis10-16. However it is not known what effect this pathway has on the global gene expression of endothelial cells. We found that constitutively active beta-catenin was sufficient to induce a small subset of the CNS endothelial transcriptional program on peripheral endothelial cells, including induction of Pgp and inhibition of Plvap. We only observed a small induction of Cldn5 and no effect on Glut1 expression. One possibility for the discrepancy is that $\mathrm{Wnt} /$ beta-catenin signaling has a greater effect on the induction of BBB gene expression during development than on the maintenance. Indeed both turning on Wnt/beta-catenin signaling and turning down Wnt/ beta-catenin signaling is important for the proper formation of CNS vessels39. Another possibility is that although Wnt/beta-catenin signaling is necessary for a wide swath of BBB-specific gene expression, it is not sufficient to induce many of these genes in peripheral endothelial cells without a second signal. We did not find that the BBBdysfunction module in peripheral endothelial cells was suppressed by activated beta-catenin signaling, suggesting that although this signal is important for development and maintenance of the $\mathrm{BBB}$, loss of this signal is not the only factor in altering brain endothelial gene expression during disease.

It should be noted that although we have achieved high levels of endothelial enrichment, we cannot exclude the fact that transcripts from other cell populations may be identified through RNA transfer, cell adherence or impurities in the preparations. This is particularly important for the disease models, in which there is extensive cellular damage and many cells taking up debris from other cell populations. Therefore, it is critical to verify that any observed changes are indeed coming from alterations in CNS endothelial cells, and not interacting cell populations.

In conclusion, we have developed an RNA sequencing resource to understand the transcriptional program of CNS endothelial cells during health and disease. This resource may provide vital information for the mechanisms by which CNS endothelial cells form their unique properties, how these properties are disrupted during injury and disease, and investigating novel targets to modulate the BBB as well as deliver drugs across the BBB to treat different neurological diseases. 


\section{Methods}

\section{Mice}

Rosa-tdTomato were bought from Jackson Laboratories (stock 07909), Rosa-Bcat-GOF were generated by Makoto Takato, and VE-Cadherin-Cre ERT2 $_{2}$ mice were generated by Ralf Adams. All experiments were performed under IACUC approval at UCSF and UCSD, and housed in a 12 hour light/dark cycle in cages of 2-5 mice. VE-Cadherin-Cre ERT2 $_{2}$ mice were originally in FvB background, and mated to Rosa-tdTomato in C57BL/6. Disease models were performed on $\mathrm{F} 1$ generation, except for EAE which was done after mating VECadherin-Cre ERT2 6 generations into C57BL/6.

\section{Disease Models}

All disease models were performed on Rosa-tdTomato; VE-Cadherin-Cre ERT2 $_{2}$ mice for endothelial cell purification and wild type C57BL/6 mice for histological analysis. Ten days prior to disease induction, Rosa-tdTomato; VE-Cadherin-Cre ERT2 $_{2}$ mice were injected 100ul of $20 \mathrm{mg} / \mathrm{ml}$ tamoxifen solubilized in corn oil for three consecutive days to induce expression of the tdTomato transgene. Males were used for MCAO, TBI and seizure, whereas females were used for EAE. Disease models were performed on mice 2-3 months of age, except for TBI as noted below.

Seizure: Mice were IP injected with $20 \mathrm{mg} / \mathrm{kg}$ kainic acid (KA) to induce seizures. Severity of seizures were scored $0-4$ based on observed behaviors: 1-freezing only; 2-freezing and occasional clonus only, 3-repeated and/or prolonged clonus, rearing and falling, 4-jumping and/or death. Mice that scored level 3 were used for brain endothelial cell isolation or tissue section immunolabeling at three different timepoints post KA injection: 3 hours (acute), 48 hours (subacute) and 1 month (chronic). For RNA sequencing experiments, 3 biological replicates were used for control and each timepoint in which each biological replicate examined was from endothelial cells enriched from the forebrain of one mouse.

Permanent MCAO: To mimic stroke, we used a permanent focal cerebral ischemia model in mice as previously described by others 40,41 . The permanent focal cerebral ischemia was induced by coagulation of the distal portion of the left middle cerebral artery (MCA).40,41 Briefly: Mice were anesthetized with a $2.5 \%$ isoflurane. The left common carotid artery was isolated and temporary ligated using 6-0 surgical Nylon monofilament suture. Mice were then placed in the lateral position, and a 2-mm bar hole was made by using a dental drill between the left orbit and ear. The distal portion of the left MCA was exposed and coagulated using a small vessel cauterizer (Fine Science Tools, Inc, CA) followed by transection of the artery. The ligation of the common carotid artery was released after 30minute occlusion. Rectal temperature was monitored and maintained at $37 \pm 0.5^{\circ} \mathrm{C}$ using a thermostat-regulated heating pad. Sham-operated mice underwent the identical surgical procedure including exposure of the common carotid artery and left middle cerebral artery except the coagulation of the distal middle cerebral artery and the temporary ligation of the common carotid artery. Tissues were harvested at 24 hours (acute), 72 hours (subacute) and 1 month (chronic) after MCAO. For RNA sequencing experiments, 3 biological replicates were used for control and each timepoint in which each biological replicate examined was 
from endothelial cells enriched from the ipsilateral region of one mouse. For dissections, the infarct region was dissected based on coordinates generated from the leakage analysis on the subacute timepoint (Fig. 2).

EAE: EAE was induced by injecting the $\mathrm{MOG}_{35-55}$ peptide containing emulsion from Hooke laboratories. Briefly, on the first day, mice were injected subcutaneously with the $\mathrm{MOG}_{35-55}$ peptide mixed with complete Freud's adjuvant (EK-2110) and simultaneously $100 \mathrm{uL}$ of Pertussis toxin was injected intraperitoneally. On the next day, the Pertussis toxin injection was repeated. Control mice were subjected to a similar protocol however the MOG peptide was not included in the Freud's adjuvant. Mice were then scored for EAE symptoms, appearance and weighed daily. Acute timepoints were taken on the first day that mice displayed a loss of 1 gram body weight. Subacute timepoints were taken after the disability score levelled off for one day. Chronic timepoints were taken two weeks after peak disease score. For RNA sequencing experiments, 3 biological replicates were used for control and each timepoint in which each biological replicate examined was from endothelial cells enriched from the spinal cord of one mouse.

TBI: The controlled cortical impact model of pediatric traumatic brain injury (TBI) was performed as previously described42. TBI to the post-natal day 21 (p21) mouse approximates a toddler-aged child based upon the structural, biochemical and behavioral characteristics of this age 43. Pups were weaned at $\mathrm{p} 21$ and anesthetized with $1.25 \%$ 2,2,2tribromoethanol (Avertin; Sigma-Aldrich, St. Louis, MO) in isotonic saline via intraperitoneal injection at $0.03 \mathrm{~mL} / \mathrm{g}$ body weight. After craniotomy, mice were subjected to a controlled cortical impact injury at $4.5 \mathrm{~m} / \mathrm{s}$ velocity and $1.73 \mathrm{~mm}$ depth of penetration, for a sustained depression of $150 \mathrm{~ms}$, using a $3.0 \mathrm{~mm}$ convex impactor tip42. Mice were maintained on a water-circulating heating pad throughout surgery and recovery. Following impact, the scalp was closed with sutures and each animal administered $0.5 \mathrm{ml}$ of isotonic saline subcutaneously to prevent post-operative dehydration. Sham-operated mice only received the Avertin injection, without surgery. Mice were weighed post-surgery at days 1, 3, and weekly thereafter. A weight loss greater than $15 \%$ per week was criteria for euthanasia; however no mice fell within this guideline. Tissues were harvested at 24 hours (acute), 72 hours (subacute) and 1 month (chronic) following TBI. Because this model was a pediatric TBI, we utilized controls for each timepoint as the mice were at substantially different stages of development across timepoints. For RNA sequencing experiments, we used 3 biological replicates for the TBI acute, subacute and chronic timepoints, and 3 biological replicates for the control-acute timepoint, and 2 biological replicates for the control-subacute and controlchronic timepoints. Each biological replicate examined was from endothelial cells enriched from one mouse. For dissections, the injured region was dissected based on coordinates generated from the leakage analysis on the subacute timepoint (Fig. 2).

\section{Immunostaining}

To generate tissues for immunostaining, mice were transcardially perfused with DPBS to remove blood, then perfused with 4\% paraformaldehyde in PBS, the tissues were dissected and cryopreserved in 30\% sucrose, frozen in 2:1 30\%sucrose:OCT and then 10 micron sections were generated. For immunostaining, tissue sections were blocked/permeabilized 
with $0.1-5 \%$ Triton X-100 and 10-50\% goat serum in PBS. Tissue sections were incubated in primary antibodies overnight at $4{ }^{\circ} \mathrm{C}$ and secondary antibodies for 1.5 hours at room temperature with $3 \%$ goat serum, $0-0.1 \%$ Triton X-100 in PBS. Primary antibodies (1/500 dilution): rat anti-mouse CD31 (clone MEC13.3, BD Pharmingen 553370), rat anti-mouse CD45 (clone YW62.3, BioRad MCA1031GA), rat anti-mouse CD140B (clone APB5, eBiosciences 14-1402-82), rabbit anti Fibrinogen (Abcam ab34269), rabbit anti Claudin 5 (Life Technologies 341600), rabbit anti Collagen I (Abcam ab21286), rabbit anti Collagen III (Abcam ab7778), rabbit anti Decorin (Biomatik CAC07220), rabbit anti Lumican (Biomatik CAU25816), rabbit anti SPP1 (Abcam ab8448). Secondary antibodies (1/1000 dilution): Life Technologies goat anti-mouse alexa-594, goat anti-rat alexa-488 and alexa-594, goat anti-rabbit alexa-594. DAPI for nuclear labeling.

\section{Biotin Permeability Assay}

For biotin leakage assays, $0.25 \mathrm{mg} / \mathrm{ml}$ sulfo-NHS-biotin dissolved in DPBS was used to transcardially perfuse mice (10 minutes, $4.5 \mathrm{ml} / \mathrm{min}$ ) in place of DPBS alone as described in the "Immunostaining" section prior to fixation with 4\% paraformaldehyde (10 minutes, $4.5 \mathrm{ml} / \mathrm{min}$ ). Biotin was visualized on cryosections with streptavidin alexa-488 (Life Technologies). Three or more mice were used for each analysis.

\section{Quantification of blood-brain barrier permeability}

Biotin permeability: Tissue sections labeled with streptavidin alexa-488 for detection of biotin were imaged and the fluorescence of alexa-488 were measured with Image $\mathbf{J}$ as pixel intensity mean gray value within a 100-pixel diameter circle region of interest (ROI). For each mouse tissue section, three ROIs were measured within the tissue areas exhibiting leakage to biotin, which corresponds to the temporal lobe in the seizure model, spinal cord lesions in EAE, infarct in the stroke model and impact in the TBI model. Meningeal labeling was not included in ROIs. The average of the three ROI measurements represent each sample. Fibrinogen permeability: The same methods were used as in biotin permeability quantification except Fibrinogen was visualized with Fibrinogen antibody and alexa-594 conjugated secondary antibody.

\section{Endothelial cell enrichment}

Endothelial cells were purified from Rosa-tdTomato; VE-Cadherin-Cre ERT2 $_{\text {mice based on }}$ methods previously described44. A graphical representation of the protocol, including modifications, is found in Supplementary Figure 8 and 9. Modifications to the procedure include: Isolated cell suspensions were re-suspended in $0.5 \% \mathrm{BSA}$ and incubated with Myelin Removal Beads II for 15 minutes at $4^{\circ} \mathrm{C}$ (Miltenyi Biotec) according to the manufacturer's protocol prior to fluorescence-activated cell sorting (FACS) purification. For immunostaining, cell suspensions were blocked in IgG serum from rat (Sigma-Aldrich I8015, $1 / 100$ in $0.5 \%$ BSA DPBS) on ice for 20 minutes, washed in $0.5 \%$ BSA DPBS, resuspended in $0.5 \%$ BSA DPBS with antibody, incubated at $4{ }^{\circ} \mathrm{C}$ for 20 minutes, washed twice and re-suspended in $0.5 \%$ BSA DPBS. For negative selection of pericytes and dead cells, cells were labeled with alexa-488 conjugated rat anti-PDGFR $\beta$ (clone APB5, Novus NBP1-43349AF488), alexa-488 conjugated rabbit anti NG2 (Bioss bs-11192R-A488) or FITC conjugated rat anti mCD13 (clone R3-242, BD Pharmingen 558744) at 1/100 and 
DAPI at $0.5 \mathrm{ug} / \mathrm{ml}$. For negative selection of immune cells, cells were labelled with: FITC conjugated rat antibodies including anti-mCD11b (clone M1/70, ebioscience 11-0112-81) and anti-mCD45 (clone 30-F11, ebioscience 0451-85) at 1/100. With FACS, cells were sorted for tdTomato, excluding cells positive for FITC/A488, both FITC/A488 and tdTomato, doublets, and DAPI+ dead cells. Cells were directly sorted into 750ul of Trizol (Life Technologies). Phenol-chloroform extraction was used to isolate nucleic acids and RNA was purified with Qiagen RNeasy Micro Kit. For the "brain endothelial" samples, a collagenase and dispase digestion step was added after trituration. Each brain was incubated in $1 \mathrm{mg} / \mathrm{ml}$ collagenase, $0.4 \mathrm{mg} / \mathrm{ml}$ dispase and 625 units of DNase in $10 \mathrm{ml}$ of enzyme stock solution at $37^{\circ} \mathrm{C}, 95 \% \mathrm{O}_{2}$ and $5 \% \mathrm{CO}_{2}$ for 30 minutes. Cell sorting was conducted at the UCSF Parnassus Flow Cytometry Core using a Beckman Coulter MoFlo XDP.

\section{RNA sequencing and Bioinformatics}

RNA sequencing for health and disease samples was done with the Gladstone Genomics core. Quality of purified RNA was accessed using an Aglient bioanalyzer. cDNA was generated from full length RNA using the NuGEN Ovation RNA-Seq V2 kit which uses the single primer isothermal amplification method to deplete ribosomal RNA. After checking cDNA size and quality on the bioanalyzer; the cDNA was quantified by NanoDrop and then sheared by the Covaris S2 Sonicator to yield uniform size fragments. The NuGen Ovation V2 kit was used to ligate adapters, and for barcoding and amplification. Libraries were purified using Agencourt XP magnetic beads, quality tested with Agilent Bioanalyzer 2100, and quantified by KAPA qPCR. Libraries were pooled and sequenced on the Illumina HiSeq2500 using paired end 100 bp reads. For mapping, trimming of FASTQ format sequences was performed using FastX_Trimmer, and sequence quality control was assessed using FastQC 0.10.1. Alignment to the Ensembl reference genome $\mathrm{mm} 9$ (v67) were performed using the splice-aware aligner Tophat 2.0.11 and bowtie2 2.21 with parameters '-no-coverage-search -m 2 -a 5 -p 7.' Alignment files were sorted using SAMtools v. 0.1.19. Count tables were generated using HTSeq-0.6.1. Differential expression was analyzed using DESeq2 1.18.1. Reads were filtered using the built-in independent filtering function of DESeq2, and P-values were calculated using a negative binomial distribution as a model for expected gene expression. FDR (false discovery rate) values were calculated with the built-in function using the Benjamini-Hochberg method.

For health, enrichment in one sample compared to another was scored for genes with $>$ twofold enrichment, cpm $>5$ in the higher sample, and $\mathrm{P}$-value $<0.05$. The BBB-specificity score for each gene was calculated as $[(\log 2(\mathrm{BE}+0.1)-\log 2(\mathrm{HE}+0.1))+(\log 2(\mathrm{BE}+0.1)-\log 2(\mathrm{KE}$ $+0.1))+(\log 2(\mathrm{BE}+0.1)-\log 2(\mathrm{LuE}+0.1))+(\log 2(\mathrm{BE}+0.1)-\log 2(\mathrm{LiE}+0.1))] 3 \times \mathrm{BE} ; \mathrm{BE}$, brain endothelial cpm, KE, kidney endothelial cpm, LuE, lung endothelial cpm, LiE, liver endothelial cpm, Peripheral endothelial-enrichment score was calculated as [ $(\log 2(\mathrm{HE}$ $+0.1)-\log 2(\mathrm{BE}+0.1))+(\log 2(\mathrm{KE}+0.1)-\log 2(\mathrm{BE}+0.1))+(\log 2(\mathrm{LuE}+0.1)-\log 2(\mathrm{BE}+0.1))+$ $(\log 2(\mathrm{LiE}+0.1)-\log 2(\mathrm{BE}+0.1))] 3 \times$ Average $(\mathrm{HE}, \mathrm{KE}, \mathrm{LuE}, \mathrm{LiE})$. For disease, up-regulation was scored for each timepoint as $\log 2$ fold $>1.00, \mathrm{cpm}>5$ in the higher sample, and Pvalue $<0.05$, and downregulation was scored for each timepoint as $\log 2$ fold $<-0.800, \mathrm{cpm}>5$ in the higher sample, and P-value $<0.05$. A lower cutoff was selected for downregulated genes $(\log 2$ fold $<-0.800)$ than upregulated genes $(\log 2$ fold $>1.00)$ as we found there were 
more robust upregulated changes than downregulated changes. This is likely due to the fact that we dissect around the disease region but will still have obtained a mixture of vessels that are leaky and those that are unaffected. Thus, it is easier to observe larger amplitudes in genes that are upregulated in the leaky vessels that are at low levels in the unaffected vessels, than it is to see genes downregulated in the leaky vessels when they are still present at high levels in the unaffected vessels. When $\log 2=$ undefined due to a 0 value in the lower sample, it was scored as a $\log 2$ fold change $>1.00$, and enrichment was then scored if $c p m>5$ in the higher sample, and $\mathrm{P}$-value $<0.05$.

We analyzed gene co-expression relationships using the $\mathrm{R}$ statistical computing environment (http://cran.us.rproject.org). Prior to co-expression analysis, low-expressed genes (defined as transcripts that were not detected in more than one quarter [17/68] of the samples) were removed. This approach filtered out 17,077 transcripts from the original set of 37,991. We proceeded to characterize co-expression relationships using a four-step approach45. First, pairwise biweight midcorrelations (bicor) were calculated for all possible pairs of transcripts over all samples using the bicor function from the WGCNA R package46. Second, transcripts were clustered using the flashClust 2 implementation of a hierarchical clustering procedure with complete linkage and ( 1 - bicor) as a distance measure. The resulting dendrogram was cut at a height corresponding to the top $10 \%$ of pairwise correlations and all initial modules were required to contain at least 12 members. Third, initial modules were summarized by their module eigengenes, defined as the first principal component obtained by singular-value decomposition of the co-expression module46. Fourth, highly similar modules were merged if the correlations of their module eigengenes exceeded a threshold (0.85). This procedure was performed iteratively such that the pair of modules with the highest correlation $(>0.85)$ was merged first, followed by recalculation of module eigengenes, followed by recalculation of all correlations, until no pairs of modules exceeded the threshold. The WGCNA measure of intramodular connectivity $\left(k_{\mathrm{ME}}\right)$ was then calculated for each transcript with respect to all modules by correlating its expression pattern across all samples with each module eigengene 47 .

\section{Quantification of the expression of BBB dysfunction module genes}

Tissue sections labeled with alexa-488 secondary antibody for visualization of the antiCD31 antibody and alexa-594 secondary antibody for visualization of the antibody against the gene of interest (GOI: collagen I, collagen III, decorin, lumican or Spp1) were imaged and the total length of vessels positive for alexa-488 or alexa-594 fluorescence were measured with the Image $\mathbf{J}$ line tool. The areas analyzed correspond to the temporal lobe in the seizure model, spinal cord lesions in EAE, infarct in the stroke model and impact in the TBI model. Meningeal vessels were not included in measurements. The percent length of vessels expressing the GOI was calculated from the total CD31 positive vessels for each tissue section.

\section{Activated Beta-Catenin studies}

Liver and lung endothelial cells were purified from 12-14 weeks old control (Rosa-Bcat-

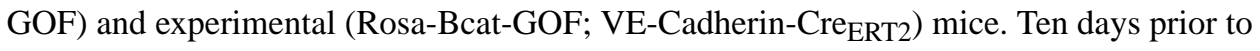
the day of endothelial cell purification, control and experimental mice were injected 100ul of 
$20 \mathrm{mg} / \mathrm{mL}$ tamoxifen solubilized in corn oil for three consecutive days to induce expression of the GOF Bcat transgene in experimental mice. Endothelial cells were purified as described in "Endothelial cell enrichment" with the following modifications: use of alexa-647-conjugated Rat mAb anti-CD31 (clone390 Molecular Probes A14716) for positive selection, omission of collagenase, dispase and myelin removal. FACS purification was done in cooperation with UCSD Center for Aids Research Flow Cytometry Core implementing a BD Aria II high speed sorter. Samples with 50,000-280,000 cells were used for RNA purification and sequencing. RNA sequencing was conducted at the UCSD Institute for Genomics Medicine Genomics Center. Method used is described in "RNA seq and Bioinformatics" with the following modifications: library generation with TruSeqRNA V2 kit and 2ng RNA input and sequencing with Illumina HiSeq4000. Sample number: 4 liver and 4 lung from 4 each control and activated beta-catenin mice. Up- and down-regulated genes due to activated beta-catenin in the liver endothelial cells, lung endothelial cells or both were identified as genes with P-value of $<0.05$ and an absolute value of $>10 \mathrm{cpm}$ in the sample with the greater value.

\section{Data Collection and Analysis}

Animals where assigned to various experimental groups at random. No statistical methods were used to pre-determine sample sizes but our sample sizes are similar to those reported in previous publications7,10,44. Disease model control and experimental tissue identity from EAE, MCAO and TBI models could not be blinded to researchers for measurement of vascular length, demarcation of region of interest and observation of tracer permeability and cellular morphology since the appearance of control and disease tissues are easily identifiable. One mouse each for TBI control acute and chronic was excluded from RNA sequencing due to signs of hemorrhage at the surface of the brain. No other data points were excluded from analyses. Information on sex, age and number of mice used for each experiment are noted in specific sections in Methods and figure legends.

\section{Statistical Analysis}

Statistical significance of differences between groups were analyzed using the following software and statistical tests: 1) DESeq2 1.18.1R Wald test (P-values) and BenjaminiHochberg method (FDR). 2) Graphpad Prism $t$ test (unpaired, parametric, equal standard deviation, two-tailed), Mann-Whitney t test (unpaired, nonparametric, two-tailed) and Friedman test (matched data, nonparametric) with post hoc Dunn's multiple comparison test. 3) DAVID Bioinformatics EASE Score (a modified one-tail Fisher Exact test). 4) Microsoft Excel $t$ test (unpaired or paired, equal standard deviation, two-tailed). Application of these statistical methods to specific experiments are noted in the figure legends.

\section{Supplementary Material}

Refer to Web version on PubMed Central for supplementary material.

\section{Acknowledgements}

We would like to thank the N. Lescano and Aaron Schroeder and the Parnassus Flow Cytometry Core, T. Rambaldo and the UCSD Veterans Hospital Flow Cytometry Core, R. Chadwick and the Gladstone Genomics Core, and A. 
Williams and The Gladstone Bioinformatics core. K. Jepsen and the UCSD Institute for Genomics Medicine Genomics Center. R.D. is funded by NIH/NINDS R01 NS091281-01A1, National Multiple Sclerosis Society Pilot Grant and Takeda Pharmaceuticals New Frontier Science Program. R.N.M. was funded by UCSF Dept. of Clinical Pharmacology and Therapeutics (GM007546) and Dept. of Anesthesia and Perioperative Care (GM008440) NIH T32 grants. M.C.O. is funded by NIH/NIMH R01 MH113896. L.J.N.-H. is funded by NIH/NINDS R01 NS050159 and NS077767. T.H. is funded by NIH/NINDS R01NS055876, NIH/NINDS R01NS082280 and Barrow Foundation Grant.

\section{REFERENCES}

1. Daneman R. The blood-brain barrier in health and disease. Ann Neurol 72, 648-672 (2012). [PubMed: 23280789]

2. Zlokovic BV. The blood-brain barrier in health and chronic neurodegenerative disorders. Neuron 57, 178-201 (2008). [PubMed: 18215617]

3. Rubin LL \& Staddon JM. The cell biology of the blood-brain barrier. Annu Rev Neurosci 22, 11-28 (1999). [PubMed: 10202530]

4. Janzer RC \& Raff MC. Astrocytes induce blood-brain barrier properties in endothelial cells. Nature 325, 253-257 (1987). [PubMed: 3543687]

5. Stewart PA \& Wiley MJ. Developing nervous tissue induces formation of blood-brain barrier characteristics in invading endothelial cells: a study using quail--chick transplantation chimeras. Dev Biol 84, 183-192 (1981). [PubMed: 7250491]

6. Armulik A. et al. Pericytes regulate the blood-brain barrier. Nature 468, 557-561 (2010). [PubMed: 20944627]

7. Daneman R, Zhou L, Kebede AA \& Barres BA. Pericytes are required for blood-brain barrier integrity during embryogenesis. Nature 468, 562-566 (2010). [PubMed: 20944625]

8. Rubin LL. et al. A cell culture model of the blood-brain barrier. J Cell Biol 115, 1725-1735 (1991). [PubMed: 1661734]

9. Weidenfeller C, Svendsen CN \& Shusta EV. Differentiating embryonic neural progenitor cells induce blood-brain barrier properties. J Neurochem 101, 555-565 (2007). [PubMed: 17254017]

10. Daneman R. et al. Wnt/beta-catenin signaling is required for CNS, but not non-CNS, angiogenesis. Proc Natl Acad Sci U S A 106, 641-646 (2009). [PubMed: 19129494]

11. Liebner S. et al. Wnt/beta-catenin signaling controls development of the blood-brain barrier. J Cell Biol 183, 409-417 (2008). [PubMed: 18955553]

12. Stenman JM. et al. Canonical Wnt signaling regulates organ-specific assembly and differentiation of CNS vasculature. Science 322, 1247-1250 (2008). [PubMed: 19023080]

13. Wang Y. et al. Norrin/Frizzled4 signaling in retinal vascular development and blood brain barrier plasticity. Cell 151, 1332-1344 (2012). [PubMed: 23217714]

14. Zhou Y \& Nathans J. Gpr124 controls CNS angiogenesis and blood-brain barrier integrity by promoting ligand-specific canonical wnt signaling. Dev Cell 31, 248-256 (2014). [PubMed: 25373781]

15. Zhou Y. et al. Canonical WNT signaling components in vascular development and barrier formation. J Clin Invest 124, 3825-3846 (2014). [PubMed: 25083995]

16. Pinzon-Daza ML. et al. The cross-talk between canonical and non-canonical Wnt-dependent pathways regulates P-glycoprotein expression in human blood-brain barrier cells. J Cereb Blood Flow Metab 34, 1258-1269, doi:10.1038/jcbfm.2014.100 (2014). [PubMed: 24896565]

17. Ioannidou S. et al. An in vitro assay reveals a role for the diaphragm protein PV-1 in endothelial fenestra morphogenesis. Proc Natl Acad Sci U S A 103, 16770-16775 (2006). [PubMed: 17075074]

18. Keuschnigg J. et al. The prototype endothelial marker PAL-E is a leukocyte trafficking molecule. Blood 114, 478-484 (2009). [PubMed: 19420356]

19. Shue EH. et al. Plasmalemmal vesicle associated protein-1 (PV-1) is a marker of blood-brain barrier disruption in rodent models. BMC Neurosci 9, 29 (2008). [PubMed: 18302779]

20. Doring A, Wild M, Vestweber D, Deutsch U \& Engelhardt B. E- and P-selectin are not required for the development of experimental autoimmune encephalomyelitis in C57BL/6 and SJL mice. J Immunol 179, 8470-8479 (2007). [PubMed: 18056394] 
21. Love S \& Barber R. Expression of P-selectin and intercellular adhesion molecule-1 in human brain after focal infarction or cardiac arrest. Neuropathol Appl Neurobiol 27, 465-473 (2001). [PubMed: 11903929]

22. Okada Y. et al. P-selectin and intercellular adhesion molecule-1 expression after focal brain ischemia and reperfusion. Stroke 25, 202-211 (1994). [PubMed: 7505494]

23. Mayadas TN, Johnson RC, Rayburn H, Hynes RO \& Wagner DD. Leukocyte rolling and extravasation are severely compromised in P selectin-deficient mice. Cell 74, 541-554 (1993). [PubMed: 7688665]

24. Huang J. et al. Postischemic cerebrovascular E-selectin expression mediates tissue injury in murine stroke. Stroke 31, 3047-3053 (2000).

25. Jin AY. et al. Reduced blood brain barrier breakdown in P-selectin deficient mice following transient ischemic stroke: a future therapeutic target for treatment of stroke. BMC Neurosci 11, 12 (2010). [PubMed: 20122276]

26. Bowes MP, Zivin JA \& Rothlein R. Monoclonal antibody to the ICAM-1 adhesion site reduces neurological damage in a rabbit cerebral embolism stroke model. Exp Neurol 119, 215-219 (1993). [PubMed: 8094342]

27. Connolly ES Jr. et al. Cerebral protection in homozygous null ICAM-1 mice after middle cerebral artery occlusion. Role of neutrophil adhesion in the pathogenesis of stroke. J Clin Invest 97, 209216 (1996). [PubMed: 8550836]

28. Henninger DD. et al. Cytokine-induced VCAM-1 and ICAM-1 expression in different organs of the mouse. J Immunol 158, 1825-1832 (1997). [PubMed: 9029122]

29. Lyck R. et al. T-cell interaction with ICAM-1/ICAM-2 double-deficient brain endothelium in vitro: the cytoplasmic tail of endothelial ICAM-1 is necessary for transendothelial migration of T cells. Blood 102, 3675-3683 (2003). [PubMed: 12893765]

30. Sharief MK, Noori MA, Ciardi M, Cirelli A \& Thompson EJ. Increased levels of circulating ICAM-1 in serum and cerebrospinal fluid of patients with active multiple sclerosis. Correlation with TNF-alpha and blood-brain barrier damage. J Neuroimmunol 43, 15-21 (1993). [PubMed: 8096220]

31. Derada Troletti C, de Goede P, Kamermans A \& de Vries HE. Molecular alterations of the bloodbrain barrier under inflammatory conditions: The role of endothelial to mesenchymal transition. Biochim Biophys Acta 1862, 452-460, doi:10.1016/j.bbadis.2015.10.010 (2016). [PubMed: 26493443]

32. Zhou T. et al. Microvascular endothelial cells engulf myelin debris and promote macrophage recruitment and fibrosis after neural injury. Nat Neurosci 22, 421-435, doi:10.1038/ s41593-018-0324-9 (2019). [PubMed: 30664769]

33. Ley K. The role of selectins in inflammation and disease. Trends Mol Med 9, 263-268 (2003). [PubMed: 12829015]

34. Conductier G, Blondeau N, Guyon A, Nahon JL \& Rovere C. The role of monocyte chemoattractant protein MCP1/CCL2 in neuroinflammatory diseases. J Neuroimmunol 224, 93 100, doi:10.1016/j.jneuroim.2010.05.010 (2010). [PubMed: 20681057]

35. Aggarwal BB, Shishodia S, Ashikawa K \& Bharti AC. The role of TNF and its family members in inflammation and cancer: lessons from gene deletion. Curr Drug Targets Inflamm Allergy 1, 327341 (2002). [PubMed: 14561180]

36. Xing C. et al. Neuronal production of lipocalin-2 as a help-me signal for glial activation. Stroke 45, 2085-2092, doi:10.1161/STROKEAHA.114.005733 (2014). [PubMed: 24916903]

37. Ferreira AC. et al. From the periphery to the brain: Lipocalin-2, a friend or foe? Prog Neurobiol 131, 120-136, doi:10.1016/j.pneurobio.2015.06.005 (2015). [PubMed: 26159707]

38. Cheng B, Chen J, Bai B \& Xin Q. Neuroprotection of apelin and its signaling pathway. Peptides 37, 171-173, doi:10.1016/j.peptides.2012.07.012 (2012). [PubMed: 22820556]

39. Ma S, Kwon HJ, Johng H, Zang K \& Huang Z. Radial glial neural progenitors regulate nascent brain vascular network stabilization via inhibition of Wnt signaling. PLoS Biol 11, e1001469, doi: 10.1371/journal.pbio.1001469 (2013). [PubMed: 23349620] 
40. Sun C. et al. Conditional ablation of neuroprogenitor cells in adult mice impedes recovery of poststroke cognitive function and reduces synaptic connectivity in the perforant pathway. $\mathrm{J}$ Neurosci 33, 17314-17325, doi:10.1523/JNEUROSCI.2129-13.2013 (2013). [PubMed: 24174664]

41. Akamatsu Y. et al. Impaired leptomeningeal collateral flow contributes to the poor outcome following experimental stroke in the Type 2 diabetic mice. J Neurosci 35, 3851-3864, doi: 10.1523/JNEUROSCI.3838-14.2015 (2015). [PubMed: 25740515]

42. Semple BD. et al. Early Gelatinase Activity Is Not a Determinant of Long-Term Recovery after Traumatic Brain Injury in the Immature Mouse. PLoS One 10, e0143386, doi:10.1371/ journal.pone.0143386 (2015). [PubMed: 26588471]

43. Semple BD, Blomgren K, Gimlin K, Ferriero DM \& Noble-Haeusslein LJ. Brain development in rodents and humans: Identifying benchmarks of maturation and vulnerability to injury across species. Prog Neurobiol 106-107, 1-16, doi:10.1016/j.pneurobio.2013.04.001 (2013).

44. Daneman R. et al. The mouse blood-brain barrier transcriptome: a new resource for understanding the development and function of brain endothelial cells. PLoS One 5, e13741 (2010). [PubMed: 21060791]

45. Lui JH. et al. Radial glia require PDGFD-PDGFRbeta signalling in human but not mouse neocortex. Nature 515, 264-268, doi:10.1038/nature13973 (2014). [PubMed: 25391964]

46. Langfelder P \& Horvath S. WGCNA: an R package for weighted correlation network analysis. BMC Bioinformatics 9, 559, doi:10.1186/1471-2105-9-559 (2008). [PubMed: 19114008]

47. Oldham MC. et al. Functional organization of the transcriptome in human brain. Nat Neurosci 11, 1271-1282, doi:10.1038/nn.2207 (2008). [PubMed: 18849986] 


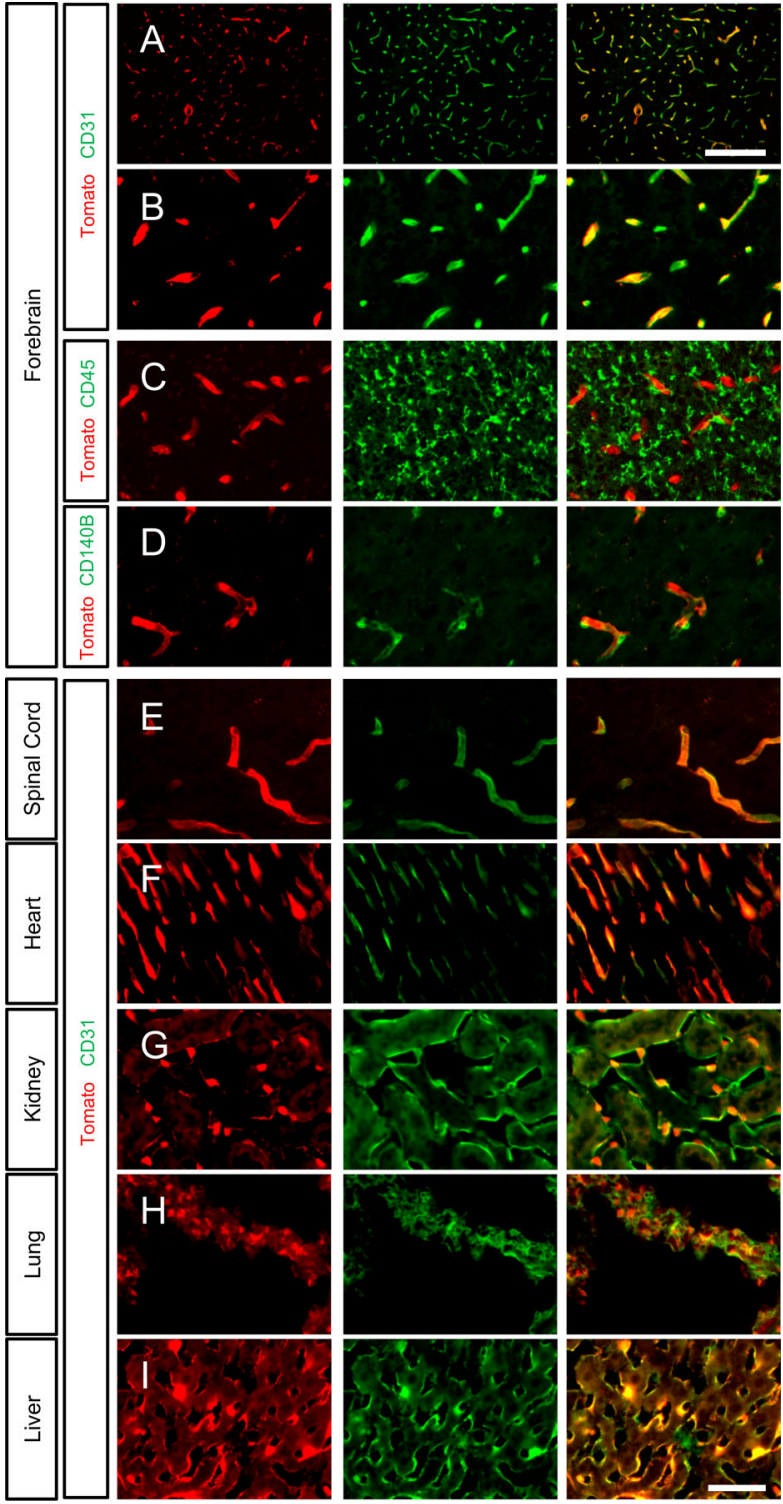

Figure 1: Endothelial reporter mouse

Tissue sections from adult Rosa-tdTomato; VE-CadherinCre ERT2 $_{2}$ mice were stained and imaged one week following a three day course of tamoxifen injections. Tissue sections from the forebrain (A-D), spinal cord (E), heart $(F)$, kidney $(G)$, lung $(H)$ and liver (I) were stained with antibodies against CD31 (green; A, B, E-I), CD45 (green, C) or CD140b (green, D). The tdTomato reporter (red, A-I) co-localized with CD31 in all tissues but not immune cells (CD45) or pericytes (CD140b). Scale bars represent 200 microns (A) and 50 microns (B-I). $\mathrm{n}=4$ mice. 


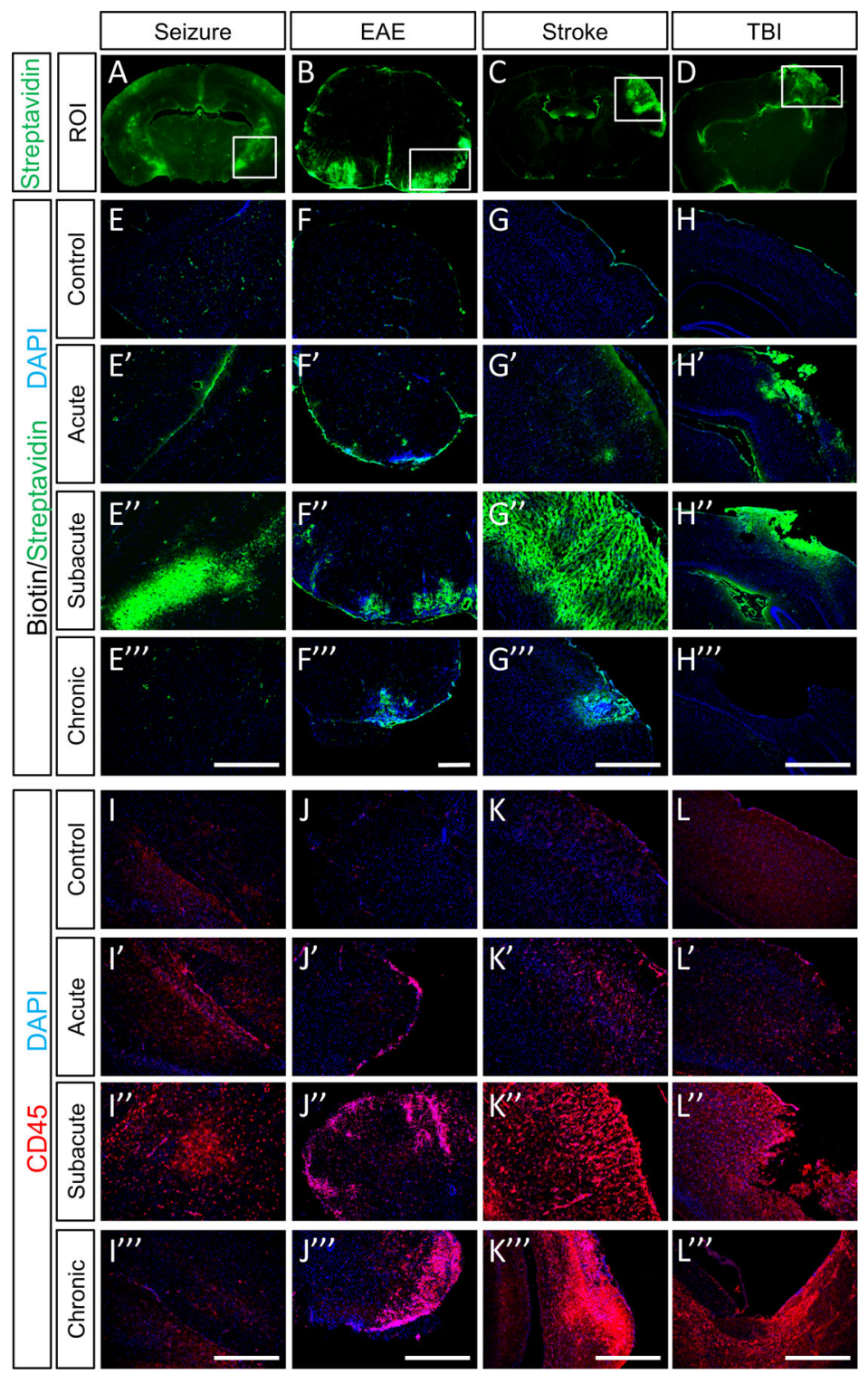

Figure 2: Blood-brain barrier leakage and inflammation following different disease models Rosa-tdTomato; VE-CadherinCre ERT2 $_{2}$ mice undergoing a kainic acid seizure model (A, E, $\mathrm{I}$,), an EAE model of MS (B, F, J), an MCAO model of stroke (C, G, K) or a pediatric TBI model (D, H, L), were analyzed at three timepoints (acute', subacute ", chronic",') for BBB leakage using a biotin tracer (green, A-H) or inflammation by staining with an antibody against CD45 (red, I-L). A-D depict low magnification images of coronal sections of the brain (A,C-D) and spinal cord (B) of biotin leakage (green) for the subacute timepoint for each disease. In images A-D the region of interest (ROI) for the disease is outlined with a white box. Subsequent images are given at higher magnification of tissue corresponding to the ROIs for controls and acute, subacute, and chronic timepoints for each disease for biotin leakage (E-H) and CD45 staining (I-L). The most BBB leakage is observed at the subacute timepoint in each disease. Scale bars represent 500 microns. n=number of mice (Control/ Acute/Subacute/Chronic): Seizure: 5/3/4/4 , EAE: 4/4/5/5, Stroke: 9/3/3/3 , TBI: 8/3/3/3. 
A Seizure EAE Stroke TBI

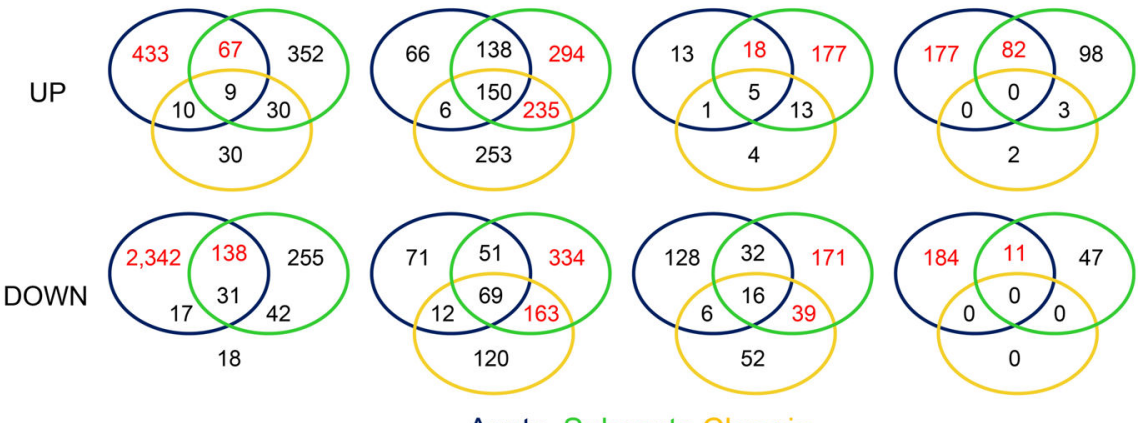

Acute Subacute Chronic

B

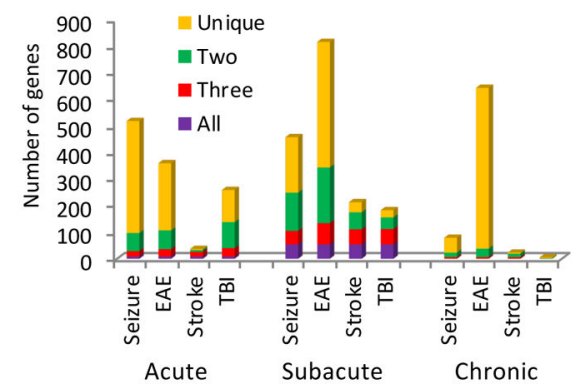

C

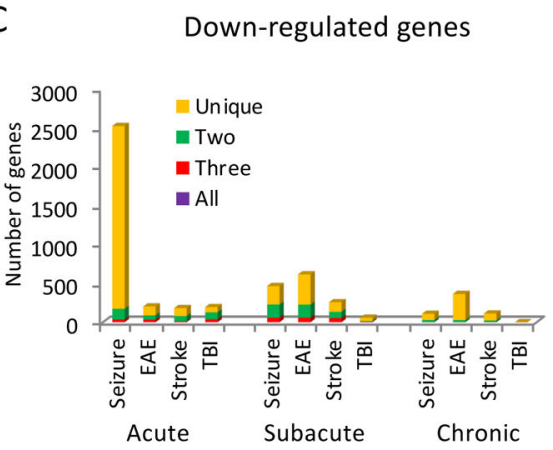

D BBB-enriched genes

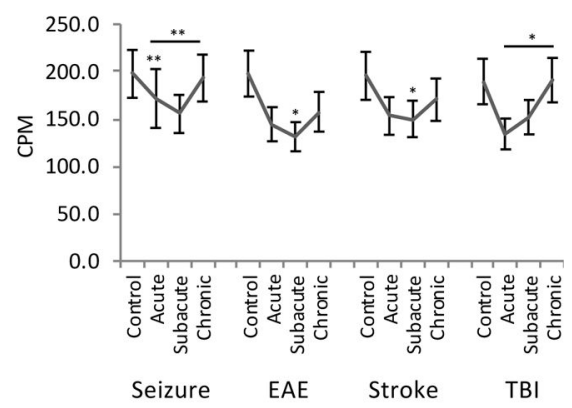

$\mathrm{E}$

Peripheral-enriched genes

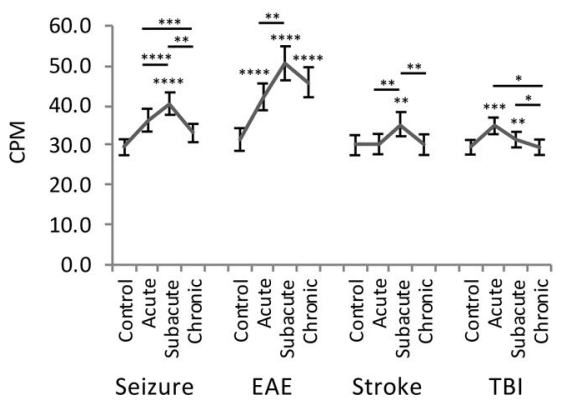

Figure 3: Cerebrovascular transcriptional changes following disease

A) Venn diagrams of the number of up-regulated (top row) and down-regulated (bottom row) gene changes in the CNS endothelial cells observed in the seizure, EAE, stroke and pediatric TBI models depicting the overlap of changes found at each of the timepoints. For each timepoint genes were selected as up-regulated if they were increased $\log 2$ fold change $>1.00$, had an expression of $>5 \mathrm{cpm}$ in the disease condition, with a P-value $<0.05$, and downregulated if they were changed $\log 2$ fold change $<-0.800$, had an expression of $>5 \mathrm{cpm}$ in the control, with a P-value $<0.05$. The timepoint with the most changes and the two timepoints with most overlap are highlighted in red. Statistical test: Wald test. $n=3$ mice each condition as source of enriched endothelial cells with exception of $n=2$ mice for TBI control subacute and chronic conditions.

B-C) Bar graphs depicting the number of gene changes at each timepoint for seizure, EAE, stroke and pediatric TBI models with color coding indicating the number of common 
changes between diseases. B) Up-regulated genes. C) Down-regulated genes. The most overlap between diseases is observed at the subacute timepoint, specifically for up-regulated genes.

D-E) The average counts per million (CPM) of all the BBB-enriched genes (D, list of genes can be found in Supplementary File 3) and peripheral endothelial-enriched genes (E, list of genes can be found in Supplementary File 5) in the CNS endothelial cells at each timepoint in the seizure, EAE, stroke and pediatric TBI models. On average there is a decrease in the expression of BBB-enriched genes and an increase in the expression of peripheral-enriched genes following each of the different disease models. Data is presented as mean $\pm \mathrm{SEM}$. Statistical test: Mann-Whitney t test (unpaired, nonparametric, two-tailed): ${ }^{*} \mathrm{P}<0.05$, $* * \mathrm{P}<0.01, * * * \mathrm{P}<0.001$ and $* * * * \mathrm{P}<0.0001$; asterisks above error bars represent comparison with control sample; horizontal lines and corresponding asterisks compare samples aligned with each end of the horizontal line. $n=518$ BBB enriched genes, 1399 peripheral enriched genes. 


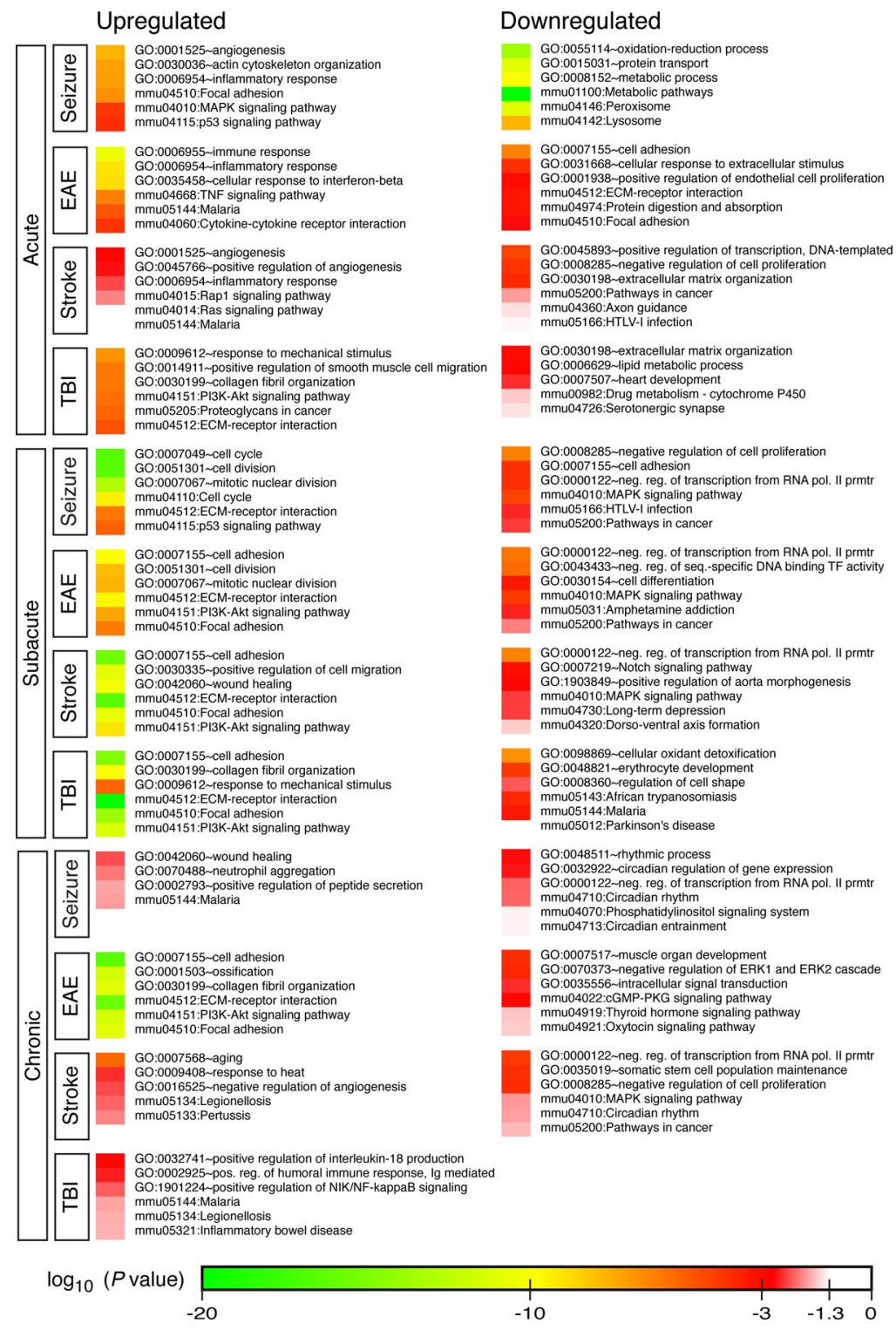

Figure 4: Pathways altered in the CNS endothelial cells following neurological disease David Bioinformatics was utilized to identify the most significantly up-regulated and downregulated GOTERMs and KEGG pathways based on the transcriptional changes in CNS endothelial cells at each timepoint for the seizure, EAE, stroke and pediatric TBI disease models. For each timepoint genes were selected as up-regulated if they were increased $\log 2$ fold change $>1.00$, had an expression of $>5 \mathrm{cpm}$ in the disease condition, with a Pvalue $<0.05$, and downregulated if they were changed $\log 2$ fold change $<-0.800$, had an expression of $>5 \mathrm{cpm}$ in the control, with a $\mathrm{P}$-value $<0.05$. The heatmap scale represents the $\log 10$ of the P-value that the specified pathway is changed in the given timepoint for each disease. The top three of each GO term and KEGG pathways are presented for each disease at each timepoint. Statistical test: EASE Score (one-tail). n=number of genes (Seizure/EAE/ Stroke/TBI): Up-regulated in Acute: 519/360/37/259, Subacute: 458/817/213/183, Chronic: 
79/644/23/5; Down-regulated in Acute: 2,528/203/182/195, Subacute: 466/617/258/58, Chronic: 108/364/113/0. 
A

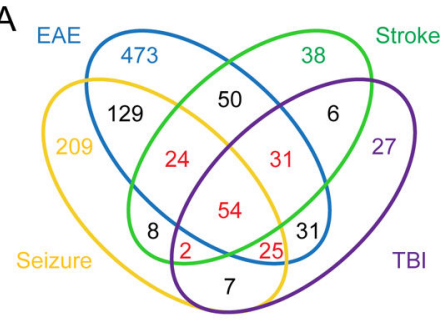

C

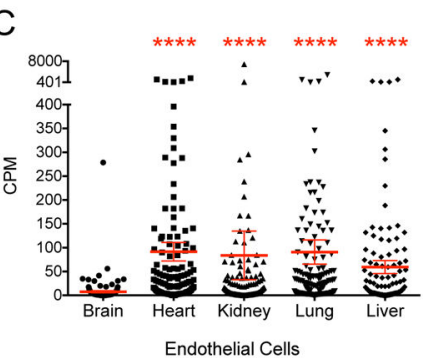

B

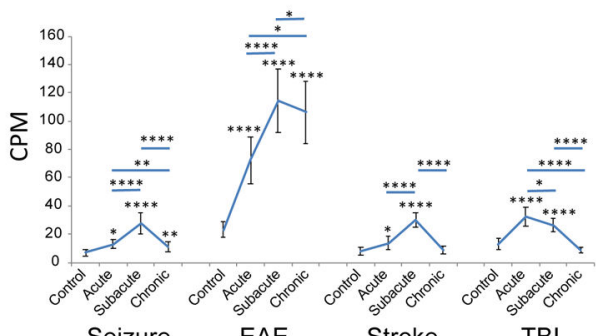

D

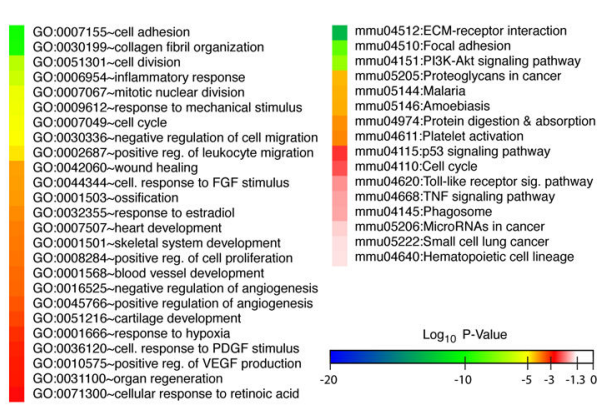

$\mathrm{E}$
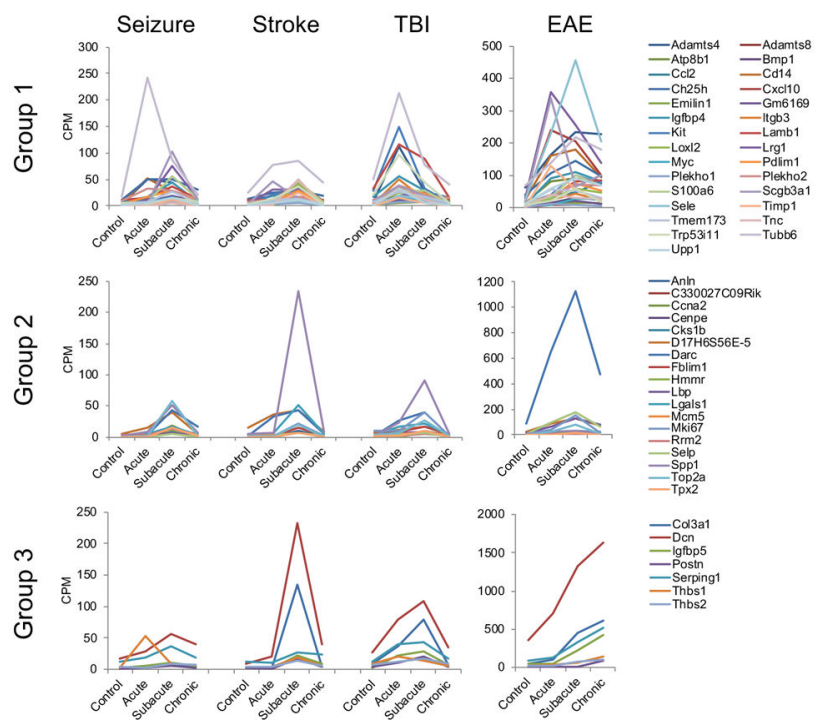

Figure 5: Identification of the BBB-dysfunction module

A) Venn diagram of the genes identified as being up-regulated in at least one of the four disease models at the subacute timepoint depicting the overlap between the diseases. Genes were considered up-regulated if they were increased $\log 2>1.00$, had an expression of $>5 \mathrm{cpm}$ in the disease sample at the subacute timepoint, with a P-value $<0.05$. The red text indicates the 136 genes up-regulated in at least three of the different disease models, that we have termed the BBB-dysfunction module (list given in Supplementary File 6). Statistical test: Wald test. $n=3$ mice each condition as source of enriched endothelial cells with exception of $\mathrm{n}=2$ mice for TBI control subacute and chronic conditions.

B) Average expression of the $136 \mathrm{BBB}$-dysfunction model genes given in counts per million (CPM), at each timepoint following the four different disease models. The BBB dysfunction module peaks at the subacute timepoint in the seizure, EAE and stroke models, and at the acute timepoint in the pediatric TBI model. Data is presented as mean \pm SEM. Statistical test: 
Mann-Whitney $\mathrm{t}$ test (unpaired, nonparametric, two-tailed): ${ }^{*} \mathrm{P}<0.05$, $* * \mathrm{P}<0.01$ and $* * * * \mathrm{P}<0.0001$; asterisks above error bars represent comparison with control sample; horizontal lines and corresponding asterisks compare samples aligned with each end of the horizontal line. $\mathrm{n}=136$ genes in each condition.

C) Average expression of the 136 BBB-dysfunction module genes in endothelial cells enriched from the brain, heart, kidney, lung and liver during health. On average, during health the BBB dysfunction module genes are greatly enriched in the peripheral endothelial cells compared to the brain endothelial cells. Data is presented as mean \pm SEM. Statistical test: Friedman test (matched data, nonparametric, result: $\mathrm{P}<0.0001$ ) with post hoc Dunn's multiple comparison (against brain sample) presented in the graph: **** $\mathrm{P}<0.0001 . \mathrm{n}=136$ genes in each organ.

D) DAVID Bioinformatics was used to identify GOTERMs and KEGG pathways that are enriched in the BBB dysfunction module. Statistical test: EASE Score (one-tail). The scale is the $\log 10$ of the EASE P-values. $n=136$ genes.

E) Average expression of the 54 BBB-dysfunction module genes up-regulated given in counts per million (CPM) in all four diseases broken down into three different groups. Group 1 reached peak up-regulation in the acute phase of many of the diseases, Group 2 reached peak expression at the subacute phase in most of the disease models, and Group 3 consisted of genes that peaked at early acute/subacute timepoints in seizure, stroke and pediatric TBI models, but continued to increase in the EAE model. 
A

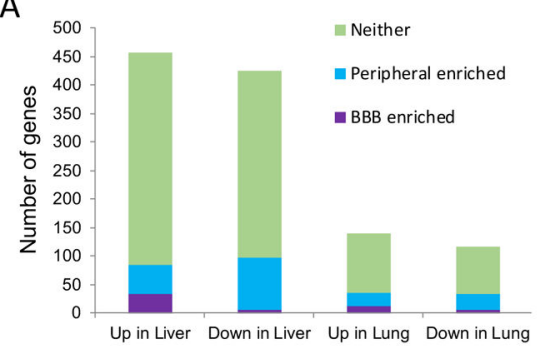

C

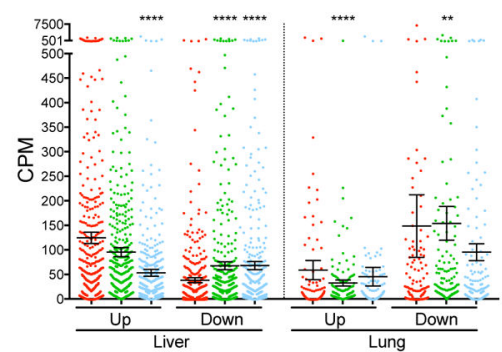

$E$
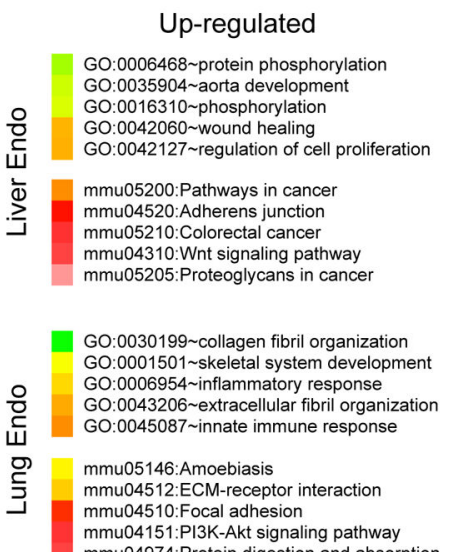

mmu04151:PIKK-Akt signaling pathway
mmu04974:Protein digestion and absorption
B

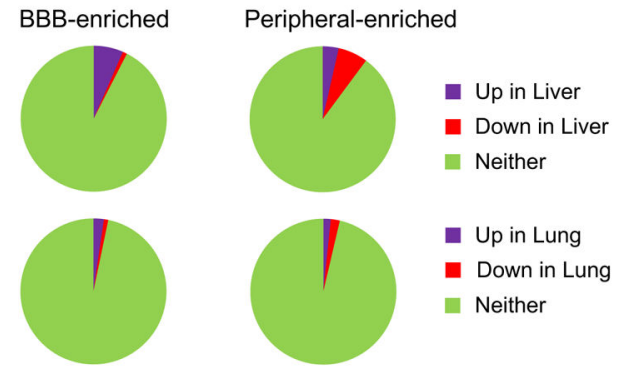

D

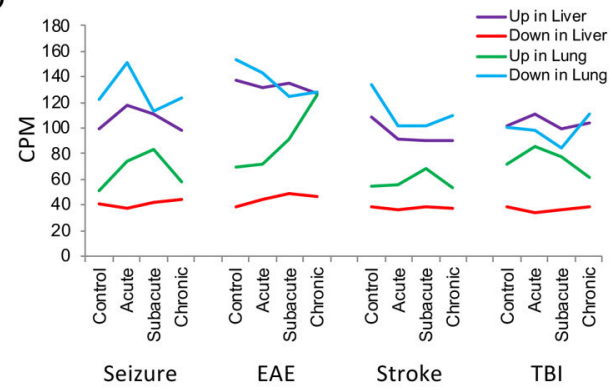

Down-regulated

GO:0055114 oxidation-reduction proces

GO:0006629 lipid metabolic process

GO:0006572 tyrosine catabolic process

GO:0006559 L-phenylalanine catabolic process

GO:0006953 acute-phase response

mmu01100:Metabolic pathways

mu01130:Biosynthesis of antibiotics

mmu00980:Metabolism of xenobiotics by cytochrome P450

mmu04610:Complement and coagulation cascades

mmu03320:PPAR signaling pathway

GO:0090316 positive regulation of intracellular protein transport GO:0001889 liver development

GO:0001889 liver developm

GO:2000121 regulation of removal of superoxide radicals

GO:0035264 multicellular organism growth

mmu00983:Drug metabolism - other enzymes

mmu04512:ECM-receptor interaction

mmu04510: Focal adhesion

$\log _{10}$ P-Value

Figure 6: Endothelial transcriptional regulation by activated beta-catenin

A) Total number of gene changes, as identified with $\mathrm{P}$-value $<0.05$ with a value of $>10 \mathrm{cpm}$ in the activated beta-catenin sample in up-regulated or control sample in down-regulated, in purified liver and lung endothelial cells from control and mice expressing activated betacatenin in endothelial cells. Genes are listed in Supplementary File 7. Genes were stratified based on whether they were identified as BBB-enriched (violet, Supplementary File 3), peripheral enriched (blue, Supplementary File 5) or neither (green). Statistical test: Wald test. $\mathrm{n}=4$ mice each condition as source of enriched endothelial cells.

B) BBB-enriched (Supplementary File 3) and peripheral-enriched (Supplementary File 5) endothelial genes were subdivided based on whether they were up- or down-regulated in liver or lung endothelial cells (Supplementary File 7) due to activated beta-catenin signaling. Statistical test: Wald test. n=518 BBB enriched genes, 1399 peripheral enriched genes. 
C) Average expression levels given in counts per million (CPM) in healthy brain (red), lung (green) or liver (blue) (CPM source, Supplementary File 3) for all genes that were up- or down-regulated in liver or lung endothelial cells to activated beta-catenin signaling (identified in Supplementary File 7). Data is presented as mean \pm SEM. Statistical test: Mann-Whitney $\mathrm{t}$ test (unpaired, nonparametric, two-tailed): ${ }^{* *} \mathrm{P}<0.01$ and $* * * * \mathrm{P}<0.0001$ represent comparison to brain sample. $n=$ number of genes: Up in Liver: 457, Down in Liver: 425, Up in Lung: 140, Down in Lung: 117.

D) Average expression levels given in counts per million (CPM) in the four disease models (CPM source, Supplementary File 1) for all genes that were up- or down-regulated in liver or lung endothelial cells due to activated beta-catenin signaling (identified in Supplementary File 7).

E) GOTERMs and KEGG pathways as identified by DAVID Bioinformatics that are regulated by activated beta-catenin in the liver and lung endothelial cells (Supplementary File 7). Statistical test: EASE Score (one-tail). The scale is the $\log 10$ of the EASE P-values. $n=$ number of genes: Up in Liver: 457, Down in Liver: 425, Up in Lung: 140, Down in Lung: 117. 